\title{
LI TZUULTAQ'A UT LI CH'OCH'. UNA VISIÓN DE LA TIERRA, EL MUNDO Y LA IDENTIDAD A TRAVÉS DE LA TRADICIÓN ORAL Q'EQCHI' DE GUATEMALA
}

\author{
Adriana C. Estrada Ochoa \\ DEP, Facultad de Filosofía y Letras, UNAM
}

A lo largo del espacio y tiempo, la tierra ha significado diversas cosas para diferentes pueblos. Para los mayas q'eqchi'es que habitan hoy en día en Guatemala, la tierra está cargada de significados muy diversos y complejos que integran elementos de su entorno natural y su realidad material, así como de los procesos históricos que han experimentado como colectividades sociales. Además, como sucede con muchos otros pueblos pertenecientes a la tradición mesoamericana, la visión de la tierra se vincula íntimamente con la identidad de los q'eqchi'es como personas y como comunidades.

El objetivo de este artículo es presentar algunos elementos que constituyen la visión q'eqchi' de la tierra a partir de un análisis de la tradición oral de este pueblo. ${ }^{1}$ El trabajo se apoya en la teoría desarrollada por Mijail Bajtín, cuyos ejes pueden servir como herramientas no sólo para el análisis literario, sino también para el estudio de realidades sociales concretas, e incluso para la construcción de una ontología.

Bajtín parte de una concepción dinámica, relacional y dialógica de la identidad. Ello significa que la construcción de la misma se realiza no sólo desde el interior de la psique como entidad aislada, sino fundamentalmente a partir de las relaciones yo-otros. Desde su perspectiva, el otro no representa un no-yo inaccesible e incomprensible, sino que es posible, en un nivel teórico, colocarse momentáneamente en su posición particular, para volver después a un nuevo sitio, un lugar de frontera. Es lo que Bajtín llama exotopía, que supone la posibilidad de conocer la perspectiva del otro, compartiendo por un momento su contexto y modo particular de percibir y experimentar la existencia y el cosmos.

\footnotetext{
${ }^{1}$ Una versión breve de este trabajo, que forma parte de mi tesis de maestría en Estudios Mesoamericanos en la Universidad Nacional Autónoma de México, fue presentada en el Vl Congreso Internacional de Mayistas, en Villahermosa, Tabasco, en 2004. Agradezco los apoyos que han hecho posible la realización de esta investigación: las becas de Conacyt y de la Dirección General de Estudios de Posgrado de la UNAM, así como el apoyo del Programa de Apoyo a Proyectos de Investigación e Innovación Tecnológica (No. IN314202), DGAPA, UNAM.
} 
La propuesta bajtiniana no implica una negación de la relación del yo consigo mismo - yo para mí-, sino una complementación de ésta con la mirada del otro. Así, en la teoría bajtiniana las relaciones identidad-alteridad no son de mutua exclusión, sino de inclusión y complementariedad.

Otro elemento de la propuesta bajtiniana que es pertinente resaltar y está íntimamente relacionado con el anterior, es el de los entimemas, es decir, los entendimientos compartidos o sobrentendidos culturales que, aunque no se dicen de manera explícita, "viven" y "circulan" en el interior de una comunidad. Los entimemas otorgan sentido a lo que se dice, dando cuenta de toda una serie de valoraciones y ordenamientos sociales de una comunidad, por lo que su conocimiento resulta fundamental para comprenderla.

Considero que los ejes planteados por la teoría bajtiniana constituyen una interesante aportación para las ciencias, ya que implican la necesidad - la exigencia incluso- de cuestionar nuestra visión del mundo a partir de un conocimiento de la visión del otro. El pensamiento bajtiniano aplicado a los estudios culturales y sociales ofrece al investigador la posibilidad de presentar diferentes visiones del mundo, incorporando las diferentes voces de los actores presentes en un contexto sociocultural determinado. Es una posibilidad para trabajar en el difícil intento de hacer consciente nuestra propia visión del mundo, cuestionarla e incluso modificarla. A partir del viaje exotópico podemos, como tradición cultural, ponernos en la posición del otro por un momento y enriquecer nuestra visión original.

\section{Ensayando el viaje exotópico:}

\section{breves apuntes sobre el mundo q'eqchi' de Guatemala}

Los q'eqchi'es constituyen uno de los más de veinte pueblos mayas contemporáneos que habitan hoy en día en Guatemala, con una población total que sobrepasa el medio millón de personas. ${ }^{2}$

Hasta antes de la segunda mitad del siglo xix el territorio q'eqchi' estaba circunscrito por los ríos Polochic y Cahabón, con grupos de población concentrados principalmente en los alrededores de Cobán, Carchá y Chamelco, así como de Cahabón y Lanquín. A partir de entonces, sobre todo desde los setenta de ese siglo, comenzaron a producirse grandes migraciones entre los q'eqchi'es, debido principalmente a los efectos de las políticas de los gobiernos liberales y a la expansión de la agroindustria en Guatemala.

\footnotetext{
${ }^{2}$ En general existen dificultades con el manejo de datos estadísticos sobre Guatemala, ya que diferentes fuentes presentan cifras muy disímiles. Con respecto a los datos sobre población q'eqchi', algunas fuentes hablan de alrededor de 300000 (Proyecto Linguístico Francisco Marroquín, 2000: 7), mientras que otras hablan de un millón (Academia de Lenguas Mayas de Guatemala, 2000: 9).
} 
Con la introducción del café como principal producto de exportación del país — producto que sustituía a la grana cochinilla y al añil- los climas fríos y húmedos de las Verapaces se volvieron muy atractivos, multiplicándose las grandes fincas de propietarios nacionales y extranjeros. La mayor parte de la tierra de Guatemala - junto con el monopolio de servicios de vital importancia, como el suministro de electricidad, el transporte ferroviario y fluvial, el correo y el control del comercio interno y hacia el exterior- era en ese entonces y hasta antes de la segunda Guerra Mundial, propiedad de alemanes.

Los despojos de tierras, la explotación y represión política de las comunidades, así como el control efectivo de la malaria y otras enfermedades propias de la tierra caliente originaron a partir de mediados del siglo xix la expansión del territorio q'eqchi'. Este proceso también se aceleró con la guerra civil del siglo xx, cuya brutalidad azotó al país por más de tres décadas, golpeando fuertemente la región q'eqchi'.

En la actualidad el territorio linguiístico q'eqchi' incluye, además de Alta Verapaz, los departamentos de Petén e Izabal, en donde constituyen la mayoría de la población, así como algunos municipios de los departamentos de El Quiché y Baja Verapaz y parte del distrito de Toledo, en Belice.

A pesar de ser el grupo maya que ocupa el mayor territorio en Guatemala, el q'eqchi' presenta poca variación dialectal en comparación con otros idiomas - como el kiche' y el mam $^{3}$ por ejemplo-, así como el mayor porcentaje de monolingüismo. Es también el que más utiliza su idioma en forma escrita, con el mayor grado de literalidad en cualquier lengua indígena del país (Grünberg, 2003: 119).

El territorio q'eqchi' tiene características singulares: es una zona estratégica, por ser el paso entre el altiplano guatemalteco, las tierras bajas del norte y la costa caribeña. Conjuga tierras altas y bajas, grandes montañas y profundas barrancas, numerosas cuevas o siguanes, ${ }^{4}$ ríos caudalosos, vegetación exhuberante, fauna y climas diversos.

Entre las peculiaridades de la historia q'eqchi' destaca el haber sido su territorio el lugar en donde se pondría en práctica el proyecto de evangelización pacífica emprendido por fray Bartolomé de las Casas, a partir del cual la hasta entonces conocida como Tezulutlán —la temible Tierra de Guerra — fue llamada Tierra de la Verdadera Paz.

Pese a la importante presencia de los q'eqchi'es a nivel demográfico y espacial, los estudios sobre cultura y cosmovisión q'eqchi' son más bien escasos y no es fácil acceder a ellos debido a barreras que establecen la geografía y el

\footnotetext{
${ }^{3}$ La ortografía que utilizo para los nombres de las lenguas y pueblos es la que sigue la Academia de Lenguas Mayas de Guatemala, excepto cuando se trata de citas textuales, donde conservo la manera de escribirlo de cada autor.

"Siguán es el nombre local con que se conoce a las profundas cuevas y cavernas que por millares cubren el territorio de las Verapaces.
} 
idioma principalmente. ${ }^{5}$ Quizás esto se deba en gran medida a las peculiaridades geográficas e históricas del área q'eqchi, que provocaron que durante varios siglos los q'eqchi'es se mantuvieran relativamente aislados del mundo occidental.

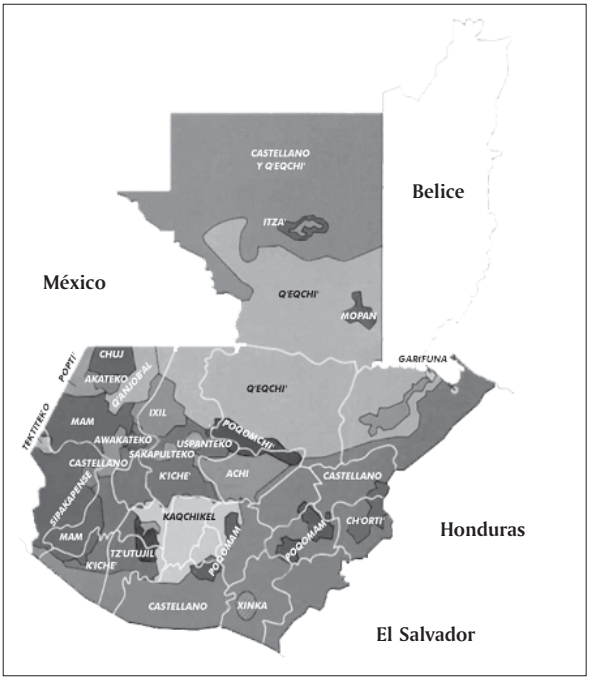

Mapa lingǘstico de GuATEmala

${ }^{5}$ Algunos de ellos, por ejemplo, sólo se han publicado en inglés y en alemán y son difíciles de encontrar. Una opción muy valiosa la constituye el excelente acervo de la biblioteca del Centro Ak' Kutan de la Orden de los Dominicos, localizada en las afueras de la ciudad de Cobán, Guatemala. 


\section{La tierra como alteridad sagrada: el Tzuultaq'a}

Uno de los entimemas que comparten muchos q'eqchi'es hoy en día, fundamental para comprender no sólo su visión de la tierra, sino también de la existencia misma, es el Tzuultaq'a. Literalmente podría traducirse como Cerro-Valle, construcción linguiística que hace referencia, más que a un espacio o a ciertos elementos del paisaje, a una alteridad central para la vida q'eqchi', que es divina y sagrada: es el Dueño de la tierra y sus riquezas, su cuidador. Cuida también los ríos, manantiales y arroyos y sus criaturas.

Tzuultaq'a es un ser vivo, cuya naturaleza conjuga ideas de dualidad y complementariedad de raíces mesoamericanas: es cerro y valle, adentro y afuera, arriba y abajo, femenino y masculino, madre y padre, uno y múltiple. Es protector y proveedor benévolo, pero también es cruel, vengativo y castigador con quienes hacen mal uso de sus riquezas o quienes no cumplen con la serie de requisitos que impone la ritualidad.

Tzuultaq'a vive en el interior de la montaña y es la montaña misma. Todo cerro es un Tzuultaq'a, pero eso no significa que todos sean iguales: dicen los q'eqchi'es que hay "trece grandes", mayores o principales, más poderosos que otros cuyo alcance es sólo local. Georg Grünberg proporciona datos sobre comunidades de Petén, en donde aunque los cerros son escasos, se reconocen trece Tzuultaq'a, así como sus correspondientes riquezas y cualidades (CARE y Cooperación Austriaca, 1999: 32):

\begin{tabular}{|c|c|}
\hline TZUULTAQ'A & Cualidades \\
\hline Qaawa' Xokob'yok & Dueño de las aves y del ganado \\
\hline Qaawa' Xucaneb' & Proporciona el agua para las siembras \\
\hline Qaawa'Raxon Tz'unum & Da la semilla de maíz o la bendice \\
\hline Qana' Itz'am & Da frijol, chile, malanga, yuca, camote y plátano \\
\hline Qaawa' Juan Kokaj & $\begin{array}{l}\text { "Coordinador", reúne a los otros doce y atiende } \\
\text { las peticiones, si están bien invocados } \\
\text { los nombres }\end{array}$ \\
\hline $\begin{array}{l}\text { Qaawa'Se’Rincón } \\
\text { Esquipuul }\end{array}$ & Recibe todas las reliquias de las mujeres \\
\hline Qaawa' Siyab' & Bendice las siembras \\
\hline Qaawa' B'ele Hu & $\begin{array}{l}\text { Fecunda la espiga del maíz y asegura que se } \\
\text { dé buena siembra, o castiga con menos cosecha }\end{array}$ \\
\hline Qaawa' Saqlech & Descubrió la semilla del maíz \\
\hline
\end{tabular}




\begin{tabular}{|l|l|}
\hline \multicolumn{1}{|c|}{ Tzuultaq'A } & \multicolumn{1}{c|}{ Cualidades } \\
\hline Qaawa' Se'llb' & $\begin{array}{l}\text { Recoge el maíz, las tortillas y otros productos } \\
\text { quemados }\end{array}$ \\
\hline Qaawa' Ton Juan & $\begin{array}{l}\text { El más pequeño de los cerros, el mensajero que } \\
\text { se encarga de encontrar el maíz }\end{array}$ \\
\hline Qana' Pet Mo' & Cerro femenino que da el frijol \\
\hline Qaawa' San Pablo & Cerro del ganado \\
\hline
\end{tabular}

A partir de los datos recopilados por diferentes autores podemos comparar los nombres de los trece Tzuultaq'a proporcionados por comunidades muy distantes, localizadas en los departamentos de Petén y Alta Verapaz. Es interesante notar que en todas las comunidades, el número trece se mantiene para referirse a los "grandes", aunque al enumerarlos muchas veces se mencionan más cerros, posiblemente por incorporar a los Tzuultaq'a locales, "patrones" de cada comunidad. El siguiente cuadro presenta algunas listas de los Tzuultaq'a elaboradas a partir de la información recogida por diferentes autores (Hernández, 2002: 15-17; Cruz, 1978: 90-9, 353-375).

\begin{tabular}{|c|c|c|c|}
\hline $\begin{array}{c}\text { Petén } \\
\text { (CARE y } \\
\text { Cooperación } \\
\text { Austriaca) }\end{array}$ & $\begin{array}{c}\text { San Pedro Carchá, } \\
\text { Cobán y Cahabón, } \\
\text { Alta Verapaz } \\
\text { (R. Wilson) }\end{array}$ & $\begin{array}{c}\text { La Tinta y Panzós, } \\
\text { Alta Verapaz } \\
\text { (O. Herrera) }\end{array}$ & $\begin{array}{c}\text { Senahú, } \\
\text { Alta Verapaz } \\
\text { (M. de la Cruz) }\end{array}$ \\
\hline Qaawa' Xokob'yok & & Qaawa' Shubyuc & Qaawa' Shucubyuc \\
\hline Qaawa' Xucaneb' & Qaawa' Xucaneb' & & \\
\hline $\begin{array}{l}\text { Qaawa' Raxon } \\
\text { Tz'unum }\end{array}$ & $\begin{array}{l}\text { Qaawa' } \\
\text { Raxuntz'unun }\end{array}$ & $\begin{array}{l}\text { Qaawa' Raxon } \\
\text { Tzunum }\end{array}$ & $\begin{array}{l}\text { Qaawa' Raxon } \\
\text { Tzunun }\end{array}$ \\
\hline Qana' Itz'am & Qana' Itz'am & Qana' Itz'am & Qana' Itzam \\
\hline Qaawa' Juan Kokaj & Qaawa' Kokaj & Qaawa' Cojaj & Qaawa' Cojaj \\
\hline \multicolumn{4}{|l|}{$\begin{array}{l}\text { Qaawa' Se'Rincón } \\
\text { Esquipuul }\end{array}$} \\
\hline Qaawa' Siyab' & Qaawa' Siyab' & Qaawa' Siab & Qaawa' Siab \\
\hline Qaawa' B'ele Hu & & Qaawa' Belebjú & Qaawa' Belebjú \\
\hline Qaawa' Saqlech & & & \\
\hline
\end{tabular}




\begin{tabular}{|c|c|c|c|}
\hline $\begin{array}{c}\text { Petén } \\
\text { (CARE y } \\
\text { Cooperación } \\
\text { Austriaca) }\end{array}$ & $\begin{array}{c}\text { San Pedro Carchá, } \\
\text { Cobán y Cahabón, } \\
\text { Alta Verapaz } \\
\text { (R. Wilson) }\end{array}$ & $\begin{array}{c}\text { La Tinta y Panzós, } \\
\text { Alta Verapaz } \\
\text { (O. Herrera) }\end{array}$ & $\begin{array}{c}\text { Senahú, } \\
\text { Alta Verapaz } \\
\text { (M. de la Cruz) }\end{array}$ \\
\hline \multicolumn{4}{|l|}{ Qaawa' Se'llb' } \\
\hline \multicolumn{4}{|l|}{ Qaawa' Ton Juan } \\
\hline \multicolumn{4}{|l|}{ Qana' Pet Mo' } \\
\hline \multicolumn{4}{|l|}{ Qaawa' San Pablo } \\
\hline & Qaawa' Tulux & & \\
\hline & Qaawa' Chiaax & & \\
\hline & & Qaawa' Cha & \\
\hline & & & Qaawa' Chirix \\
\hline & & & Quiché \\
\hline & Qaawa' Chinam & & \\
\hline & & Qaawa' Chajcoj & Qaawa' ChajCoj \\
\hline & & Qaawa' Tzunkin & Qaawa' Tzunkim \\
\hline & Qaawa' Raxon & & \\
\hline & & Qaawa' Tac'caj & Qaawa' Tac' Caj \\
\hline & Qaawa' Saqipek & & \\
\hline & & Qaawa' Chajmaic & Qaawa' Chajmaic \\
\hline & & Qaawa' Chijaal & Qaawa' Chijaal \\
\hline & & Qaawa' Cubilgüitz & Qaawa' Cubilgüitz \\
\hline & Qaawa' San Vicente & & \\
\hline & & $\begin{array}{l}\text { Qaawa' } \\
\text { Chisguajagua }\end{array}$ & $\begin{array}{l}\text { Qaawa' } \\
\text { Chisguajagua }\end{array}$ \\
\hline & & & Zac Tzicnil \\
\hline & Qaawa' Ixim & & \\
\hline & Qaawa' Sa'Mastun & & \\
\hline
\end{tabular}


Si bien las variaciones en los nombres de los "trece grandes" son considerables, no debemos menospreciar las coincidencias, que posiblemente se explican debido a que, según han sido identificados, muchos de estos cerros se encuentran localizados en el "corazón" original del mundo q'eqchi'. ${ }^{6}$

Los Tzuultaq'a, como sus hijos los q'eqchi'es, se relacionan entre sí en términos de parentesco, desempeñando papeles familiares y comunitarios. Darío Caal porporciona la siguiente clasificación (Caal, 1996: 59):

El padre Xucaneb'
La hija Suqk'im
La anciana consejera Qana' Ab'as
El anciano cerbatanero Qaawa' Saq Lech'
El yerno Qaawa' K'ix Mes
El flechero Qaawa' Tzimaj T'ok'
Los vecinos Qaawa' Chi Chen,
Qaawa' Razón,
Qaawa' Kokaj,
Qaawa' Siyab'

Hay un actor —o sería más apropiado decir actriz - que no aparece en este esquema reportado por Caal y que desempeña una serie de papeles centrales en términos sociales: la mujer Tzuultaq'a, esposa de Xucaneb' y madre de Suqk'im, según algunos relatos de tradición oral ("Sucesos remotos", 1996: 6874), o amante de Qawa' Siyab' y madre de Ixckkaaliil en otros (Curley, 1980: 9397). Se trata de Q'ana Itzam, "la vieja madre de los Tzuultaq'a”, que aparece como protectora feroz y aguerrida de sus criaturas en la narrativa oral (Cruz, 1978; Schakct, 1986). Es también la madre de Qawa' Ixim, "Señor Maíz, de agradable talante, muy querido y reverenciado por todos" (Dieseldorff, 1984: 3).

Los Tzuultaq'a conversan entre ellos por medio del viento y de bolas de fuego (fuegos fatuos) que ocasionalmente se ven salir de un cerro y volar hacia otro (Pacheco, 1995: 65). También tienen apetitos y pasiones. Se enamoran, como Qana' Itzam y Qawa' Siyab', o como Qawa' Bolonwinq y Qana' Ixckkaaliil, secuestrada esta última por Mauserwinq, "amo de la soberbia, del robo, del hurto y de la muerte" (Curley, 1980, 93-97). Viven romances y aventuras, como cuando Qawa' K'ix Mes secuestró a Suq K'im, hija de Xucaneb'. Tienen conflictos y pelean entre ellos, como sucedió cuando Qawa' Saqle'ch — ante el desaire de Suq K'im, que prefirió casarse con K'ix Mes- decidió esconder en su interior el maíz que Xucaneb' le había encomendado guardar ("Sucesos remotos", ibid.).

\footnotetext{
${ }^{6} \mathrm{Al}$ respecto, Pacheco menciona que algunos cerros identificados se localizan fuera del área q'eqchi', como el Raxon Tzunum, que se encuentra en la Sierra de las Minas y el Tac'aj, ubicado en el departamento de Quetzaltenango (Pacheco, 1995: 69).
} 
Los Tzuultaq'a se alimentan de las oraciones, el humo del copal pom, las candelas, el cacao, el $b o j^{7}$ y la sangre de los animales sacrificados en su nombre. Se conmueven y encolerizan con los hombres, sus hijos, a quienes pueden enviar salud y prosperidad pero también enfermedad y desgracia. Es gracias a ellos que llega la lluvia que riega las milpas q'eqchi'es, pues ellos la llaman por medio de los truenos. También la detienen para evitar desastres, por medio del arco iris que ponen en el cielo para mantenerla a raya.

\section{Tierra, mundo e identidad}

En el título del artículo hago referencia también al mundo ya que considero que, desde la visión q'eqchi', las distinciones naturaleza-mundo-cosmos no existen o por lo menos no son muy claras. Ello se manifiesta, por ejemplo, en la lengua, como sucede con la palabra $c h^{\prime} o c h$ ', que significa tierra y sirve como raíz para la construcción de otras palabras como ruuchich'och', que generalmente se traduce como mundo. Por su parte, la combinación de cielo y tierra, choxaach'och', se traduce como naturaleza, universo o cosmos.

Quizá la tierra sea, desde esta perspectiva, uno de los elementos del mundo, o bien el mundo mismo. Es, aunque suene a lugar común, más que un recurso explotable: es un espacio sagrado, que proporciona vida, alimentos y medicinas, así como refugio para vivos y muertos, a los que da cabida cuando termina su existencia. Pero no sólo eso: es también eje de identidad a nivel personal y colectivo. La tierra como alteridad es tan importante en el pensamiento indígena de tradición mesoamericana que sus elementos —el cerro, el valle, el aguaresultan indispensables para la construcción del cuerpo social y político de las comunidades. Ello significa que, siguiendo la perspectiva bajtiniana, entre las alteridades involucradas en la construcción identitaria no sólo se encuentran las de carácter étnico, sino también, y de manera central, las del mundo de lo sagrado, que desde la perspectiva q'eqchi incluye por supuesto el mundo natural.

Los q'eqchi'es son aj ral ch'och', hijos de la tierra. Es así como se conciben y se definen a sí mismos. Un informante de la aldea Sexan, municipio de El Estor, Izabal, lo explicó así:

la tierra es como nuestra madre. No nos damos cuenta de que cuando nacemos la madre nos da todo, nos alimenta, nos cuida. Igual pasa con la tierra $[\ldots]$ Nacemos aquí y morimos aquí. Ahí vamos a terminar todos nosotros. Por eso es parte de nosotros. Cuando un niño llora, la madre le da pecho. La tierra cuando nos mira llorar y pedirle de corazón, ella nos lo da. ${ }^{8}$

\footnotetext{
${ }^{7}$ El boj es una bebida tradicional que se prepara a partir de maíz y caña de azúcar fermentados.

${ }^{8}$ Informante q'eqchi' de la aldea Sexan, municipio de El Estor, Izabal, febrero de 2003.
} 
También se llaman a sí mismos roqch'och', "pie de la tierra”. ${ }^{9}$ Son tzakal winq o yal winq, que literalmente significa "verdadero hombre", "hombre auténtico", "hombre perfecto" (Pacheco, 1988: 8) Pienso que más que tratarse de una definición etnocentrista, esto hace referencia al ideal q'eqchi' acerca de la persona y la comunidad: ellos consideran que su manera de relacionarse con la tierra y el mundo son las correctas o "verdaderas", en contraposición con la manera desacralizada, instrumental y utilitaria que propone Occidente.

Podemos encontrar evidencia linguiística del vínculo entre la tierra y la identidad q'eqchi' a nivel individual, como personas: utilizan la misma palabra, ch'och' para referirse a la tierra y al hígado. También utilizan la palabra kenq, para referirse tanto a los frijoles como a los riñones. ${ }^{10}$ Quizá esto se explica debido a la semejanza en la forma de frijoles y riñones, o a la apariencia terrosa de un hígado. Pero también puede mostrar que para los q'eqchi'es, como aj ral ch'och', no existe diferencia entre la forma, la naturaleza o quizá incluso la sustancia o materia que constituye su propio cuerpo y el de la tierra y sus frutos.

A nivel comunitario también podemos encontrar evidencias lingüísticas del vínculo con la tierra: la palabra $a m a q$ ' se utiliza para designar los conceptos de compañero, pueblo y territorio. Otra evidencia, que refiere además a una idea de integración cósmica es el uso de las palabras ch'och'el y sululel para referirse tanto a la estructura física como a la patria, la tierra natal y la cultura y "raza" maya (MINUGUA, 1997).

La tierra también vincula a los q'eqchi'es con los antepasados y su sabiduría ancestral. Para referirse a los ancestros y a su tiempo, lo antiguo, utilizan un término que hace alusión al mundo vegetal: xeton, compuesta por xe, raíz y ton, tronco.

También vinculado con la tierra y los antepasados se encuentra el ch'ol winq, a quien los q'eqchi'es conciben como el antepasado maya jamás conquistado y por quien se siente una mezcla de admiración, respeto y temor. El ch'ol winq es visto como un ser "salvaje" y temerario, que cuida a los animales y que por las noches va de cacería por el monte, trayendo la perdición de quienes se cruzan por su camino. Es también poseedor de grandes e inexplicables secretos: cuentan que fueron ellos quienes contruyeron Tikal con el poder del silbido (Teni, 2003).

La tierra también es hogar de otras alteridades como el q'eq, que literalmente significa negro pero que también suele traducirse como "espanto". Éste es un personaje común en la narrativa oral mesoamericana, que ha sido interpretado como los capataces negros de las fincas coloniales, por lo que son asociados con la represión y la explotación y, por ello, el espanto.

\footnotetext{
${ }^{9}$ Otto Schumann, comunicación personal, abril de 2004.

${ }^{10}$ Esto sucede por lo menos en la variante de Cobán, que constituye el dialecto de prestigio, por lo que su uso se expande cada vez más, sobre todo entre la población más joven.
} 
En la narrativa de tradición oral constantemente aparece la tierra como alteridad sagrada. El Tzuultaq'a es en los relatos, junto con los astros, el rayo, la nube y algunos animales de comportamiento asombroso, personaje de relatos cosmogónicos que viven aventuras en un tiempo anterior al de los hombres.

Los Tzuultaq'a son también "héroes culturales" que proporcionaron a los q'eqchi'es herramientas materiales y tecnológicas para sostenerse, constituyendo sus oficios de carácter identitario: fue Qawa' Ton Juan quien otorgó el maíz a los hombres para que pudieran alimentarse, al extraerlo del interior de un cerro con el poder de su hacha o tambor, el rayo (Cruz, 1978: 81-97; Curley, op. cit.). También fueron los Tzuultaq'a quienes compartieron los secretos de la medicina con algunos iniciados, como Pablo Maquín Cajbom o Cajbon — personaje que aparece en relatos recopilados en Izabal y en Alta Verapaz-, que sabía bien cómo curar de la mordida de la terrible $i k$ b'olay o nauyaca, y de quien se dice que "tenía secretos con los señores de la montaña y por eso iban a él cuando había un enfermo que sanar" (Sahil ch'oolej sa' li hoonal, 1995: 37; Cruz Torres, 1978: 71-80).

Fue Qana' Itzam quien proporcionó los secretos del tejido a las mujeres para que pudieran vestirse y no pasaran frío (Dieseldorff, 1984: 6) y fue también ella quien "terminó con las conquistas", llamando al resto de los Tzuultaq’a para que enviaran sus fuerzas y levantando a las mujeres para que pelearan junto con los hombres en contra de los "moros" que hacían retumbar la tierra en el occidente guatemalteco (Cruz, 1978: 99-106).

\section{La tierra como tema recurrente y como discurso identitario}

Las enseñanzas morales son tema recurrente en la narrativa oral, en donde el asunto de la relación con la tierra parece casi una obsesión. Los relatos enfatizan la necesidad de volver a los valores de equilibrio, respeto y reciprocidad con la tierra y el cosmos, retomando el legado de los antepasados y reconociéndose nuevamente como aj ral ch'och' o hijos de la tierra.

Algunos se preocupan por el hecho de que se pervierta su forma de relacionarse con la tierra, como lo demuestran las palabras de nuestro informante de Sexan:

Es como si el aliento de vida se estuviera acabando. La tierra nos da de mamar de pequeños y nos sigue dando. Siempre nos está ayudando para seguir viviendo [...] Algunas personas ya no respetan las costumbres de los ancianos, los ancestros. Eso está perjudicando, que ya no se practique. El mayejak siempre se realizaba pero ahora se está perdiendo. Los jóvenes ya no lo conocen, ya no lo practican. Es muy doloroso porque nosotros vivimos de la tierra. Hemos sido golpeados 
por otras culturas, otras ideologías. Ello ha propiciado que los jóvenes pierdan el respeto. Ha cambiado su mentalidad. ${ }^{11}$

Ellos reaccionan ante esta preocupación y proponen retomar los valores de reciprocidad y respeto a la tierra, reforzando así su vínculo con ella y revitalizando su identidad. Como lo decía el mismo informante:

A mí así me lo enseñaron mis padres, mis abuelos. Así me gustaría enseñar a mis hijos y nietos. Están cambiando las mentalidades pero en cada familia puede haber alguien que sepa cómo es. A mí me enseñaron que cuando pedimos a la montaña llevamos un sacrificio, por ejemplo una gallina para darle de comer a la montaña. Es muy doloroso ver que los jóvenes ya no lo hacen. ${ }^{12}$

Se trata de una inquietud que no es nueva; Estrada Monroy publicó un documento que da cuenta de la percepción de los q'eqchi'es ante lo que proponía la alteridad kaxlan hacia el siglo xv, propuesta que negaba no sólo su visión del mundo sino también la de sí mismos (1993: 191-193). Dice así:

\section{LAMENTACIÓN Q'EQCHI'}

¡Ay Dios! Qué duro es lo que hoy decís que es otra la única verdad iAy Dios! Nuestras Madres, Nuestros Padres nos enseñaron y nosotros enseñamos a nuestros hijos y nuestras hijas, quien hizo todas las cosas. ¿Acaso no hemos sabido desde siempre cómo se formó la vida, cómo se generaron las cosas, cómo se hicieron las aguas, cómo se hizo la tierra cómo se hicieron las plantas y cómo se mejoraron las cosas?

iAy Dios! Qué duro es lo que decís que crea que ésta no es la verdad, sino otra que vos me decís.

iAy Dios! ¿Acaso no nos dijeron nuestras Madres y Nuestros Padres que Tzuul-tak'a nos daba el maíz, el agua, la lluvia, el fuego?

iAy Dios! Acaso nosotros y nuestros hijos

no hemos ofrecido el pom y golpeado nuestras

\footnotetext{
${ }^{11}$ Informante q'eqchi' de la aldea Sexan, municipio de El Estor, Izabal, febrero de 2003.

${ }^{12}$ Véase nota anterior.
} 
piernas en la cumbre del camino?

¿Acaso no tuvimos comida y no se curaron nuestros dolores?

¿Acaso no se quitó nuestro calor

y nuestro frío?

iAy Dios! qué duro es lo que decís que la verdad

es otra iAy Dios!

iAy Dios! Qué duro es tener que creer que

Tzuul-tak'a no existe, ni existió, ni existirá,

si está allí en el siguán, si está

en el cerro, si se le oye en el trueno,

si se ve su luz en el cielo

iAy Dios! Qué duro es creer que pasar a

nuestros hijos y nuestras hijas sobre

el fuego y que nuestro atol de masa

y nuestra tortilla no trae a nuestros cuerpos

el aliento de Tzuul-tak'a para que

seamos buenos.

iAy Dios! que duro es creer que todo esto

fue en vano.

iAy Dios! Qué duro es creer que nuestras Madres

y nuestros Padres, nuestros sacerdotes

no nos hablaron con la verdad.

¿Acaso no dijeron qué día era bueno y qué día

era malo?

¿Acaso no hicieron bien las ofrendas, los sacrificios, y encendieron el fuego

y quemaron el pom?

¿Acaso no se evitaron los males, acaso

nos golpeamos en el camino o caímos a

lo hondo del siguán?

iAy Dios! Qué duro es creer que

Tzuul-tak'a no nos escuchará más y

que ya no escuchará nuestro llanto.

Este documento, como los rezos, las acciones rituales y los relatos que se cuentan hoy en día, expresan la importancia y sacralidad de la tierra q'eqchi' y de las relaciones que tienen con ella como aj ral ch'och'. 


\section{BIBLIOGRAFÍA}

Aatinaqo sa' q'eqchi' mayab'. Hablemos en q'eqchi'

2000 Academia de Lenguas Mayas de Guatemala. Guatemala: Paxil.

Bajtín, Mijail

1982 Estética de la creación verbal. México: Siglo XXI.

2000 Yo también soy. Fragmentos sobre el otro. Prol. Tatiana Bubnova. México: Taurus.

Caal, Darío "Mayejak", Voces del tiempo, 19: 58-63. Guatemala: Ak'Kutan. 1996

Cruz Torres, Mario Enrique de la

1978 Rubelpec. Cuentos y leyendas de Senahú, Alta Verapaz. Guatemala: Editorial del Ejército.

Curley García, Francisco

1980 Ma Yan Pan. Civilización y cultura gkec-chí. Guatemala: Tipografia Nacional.

Chacach, Martín

1995 "La comunidad lingüística q'eqchi' ", Prensa Libre, Guatemala, 17 de junio.

Diccionario Q'eqchi' Molob'aal Aatin

2000 La Antigua: Proyecto Linguiístico Francisco Marroquín.

Dieseldorff, Herbert Quirín

1984 X Balam Q'ué, el Pájaro Sol. El traje regional de Cobán. Guatemala: Ediciones del Museo Ixchel.

Estrada Monroy, Agustín

1979 El mundo K'ekchí de la Verapaz. Guatemala: Editorial del Ejército.

1993 Vida esotérica Maya-K'ekchí. Guatemala: Serviprensa.

Grünberg, Georg

2003 Tierras y territorios indígenas en Guatemala. Colección Dinámicas Agrarias VI. Guatemala: FLACSO, MINUGUA, CONTIERRA.

Hernández Tohom, Jesús Trinidad

2002 'Aj ral ch'och xnaleb'eb' aj q'eqchi' re Peten. El hijo de la tierra. Sabiduría de los q'eqchi'es de Petén. Experiencia Pastoral". Tesis de licenciatura en Teología. Guatemala: Universidad Rafael Landivar.

Manual de Comunidades de Petén

1999 Guatemala: CARE y Cooperación Austriaca. 
$1997 X$ Xtusalal ru aatin chi rix xna'leb'il li xk'ulub'eb' li poyanam kaxlan aatin -q'eqchi', Vocabulario Español-Q'eqchi' con relación a los derechos humanos. Guatemala: Oficina Regional en Cobán.

Pacay, Eduardo

1989 "Aproximación a la cosmovisión maya y la concepción k’ekchí del $M u^{\text {", en }}$ Memorias del II Coloquio Internacional de Mayistas, pp. 897-904. México: UNAM, IIFL, Centro de Estudios Mayas.

Pacheco, Luis

1988 Tradiciones y costumbres del pueblo maya kekchí. Noviazgo, matrimonio, secretos, etc. San José, Costa Rica: Editorial Ámbar.

1995 La religiosidad contemporánea Maya-Kekhí. Quito: Abya-Yala.

Sahil ch'oolej sa' li hoonal (Momentos alegres). Leyendas q'eqchi'es de El Estor, Izabal 1995 Rancho Palos Verdes, California: Ediciones Yax Te'.

Schackt, John

1986 One God-Two Temples. Schismatic process in a Kekchi Village. Oslo: University of Oslo (Occasional Papers in Social Anthropology).

Sedat, Guillermo

1971 Nuevo diccionario de las lenguas k'ekchi' y española. Guatemala: Instituto Linguiístico de Verano.

Siebers, Hans

1996 "Creolization and modernization at the periphery: the case of the Q'eqchi'es of Guatemala". Tesis doctoral. Holanda: Universidad Católica de Nijmegen.

Ramazzini, Álvaro et al. (eds.)

1996 Teología india mayense II. Memorias, experiencias y reflexiones de encuentros teológicos regionales (junio). Guatemala: CENAMI.

Teny Maquín, César A.

2003 "Modelo pedagógico maya". Tesis de licenciatura en Pedagogía. Guatemala: Universidad Rafael Landívar.

Vocabulario q'eqchi', Xtusalal aatin sa' q'eqchi' 2001 Guatemala: Academia de Lenguas Mayas de Guatemala.

Wilson, Richard

1999 Resurgimiento maya en Guatemala (experiencias Q'eqchi'es). La Antigua: Centro de Investigaciones Regionales de Mesoamérica. 